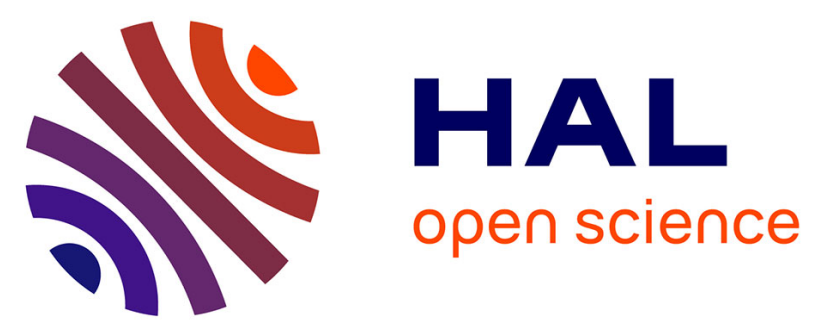

\title{
Experimental and theoretical study on the capture/desorption of gaseous methyl iodide on sea salt aerosols
}

Maxime Infuso, Hanaa Houjeij, Céline Toubin, Anne Cecile Gregoire, Gwenaelle Le Bourdon, Laurent Cantrel, Sonia Taamalli, Florent Louis, Denis Duflot, Sophie Sobanska

\section{To cite this version:}

Maxime Infuso, Hanaa Houjeij, Céline Toubin, Anne Cecile Gregoire, Gwenaelle Le Bourdon, et al.. Experimental and theoretical study on the capture/desorption of gaseous methyl iodide on sea salt aerosols. European Geoscience Union - General Assembly EGU 2020, May 2020, EN LIGNE, France. hal-03107892

\section{HAL Id: hal-03107892 \\ https://hal.science/hal-03107892}

Submitted on 12 Jan 2021

HAL is a multi-disciplinary open access archive for the deposit and dissemination of scientific research documents, whether they are published or not. The documents may come from teaching and research institutions in France or abroad, or from public or private research centers.
L'archive ouverte pluridisciplinaire HAL, est destinée au dépôt et à la diffusion de documents scientifiques de niveau recherche, publiés ou non, émanant des établissements d'enseignement et de recherche français ou étrangers, des laboratoires publics ou privés. 

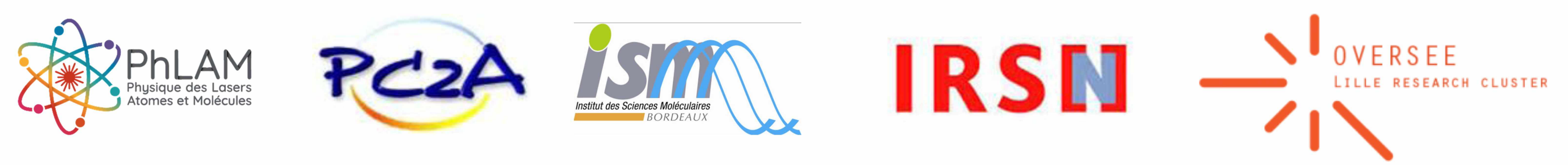

\title{
Experimental and theoretical study on the capture/desorption of gaseous methyl iodide on sea salt aerosols
}

\author{
Hanaa Houjeij ${ }^{1,2}$, Maxime Infuso ${ }^{3,4}$, Anne-Cécile Gregoire2, Gwenaelle Le Bourdon ${ }^{1}$, Laurent Cantrel2, Sonia \\ Taamalli $^{3}$, Florent Louis ${ }^{3}$, Denis Duflot ${ }^{4}$, Céline Toubin ${ }^{4}$ and Sophie Sobanska ${ }^{1}$ \\ ${ }^{1}$ Institut des Sciences Moléculaires, Université de Bordeaux, 33405, Talence, France \\ 2 Institut de Radioprotection et de Sûreté Nucléaire, PSN-RES, 13115, Saint Paul Lez Durance cedex, France \\ ${ }^{3}$ Laboratoire PC2A, Université de Lille, 55655 Villeneuve d'Ascq Cedex, France \\ ${ }^{4}$ Laboratoire PhLAM, Université de Lille, 55655 Villeneuve d'Ascq Cedex, France
}




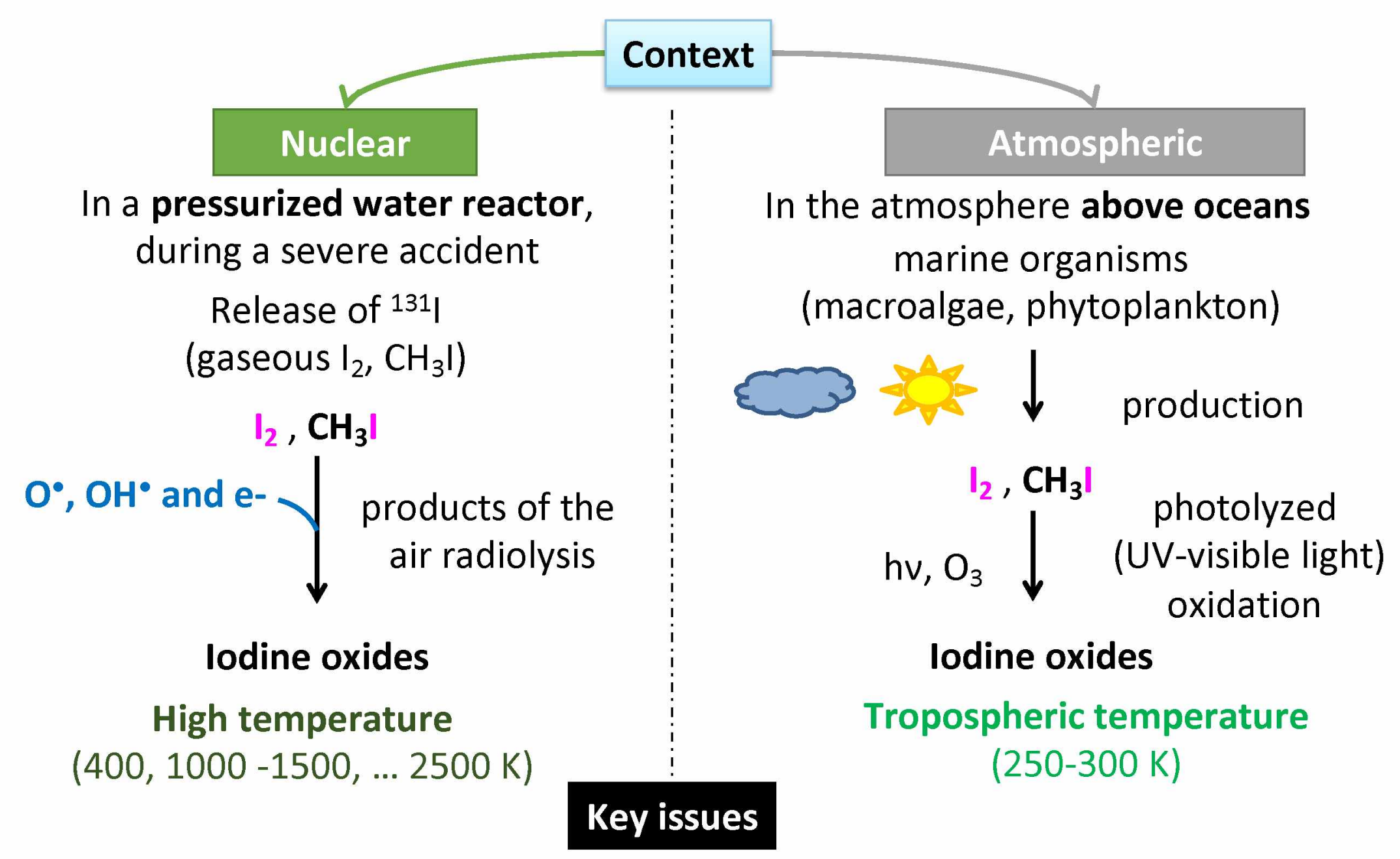

How the methyl iodide reactivity is influenced by its environment?

$\rightarrow$ Reactions with atmospheric aerosols ?

$\rightarrow$ Influence of the presence of $\mathrm{H}_{2} \mathrm{O}$ ? 


\section{Diffuse Reflectance Infrared Fourier Transform Spectroscopy (DRIFT) experiments}

$\checkmark$ To probe Chemical and structural information for solid surfaces exposed to gaseous species

$\checkmark$ Provide semi quantitative analyses for kinetics, adsorption-absorption and surface reaction

$\checkmark$ Reproduce atmospheric conditions $(\mathrm{P}, \mathrm{T})$

$\checkmark$ FTIR spectra reprocessing in Pseudo absorbance ( $\log (1 / R))$

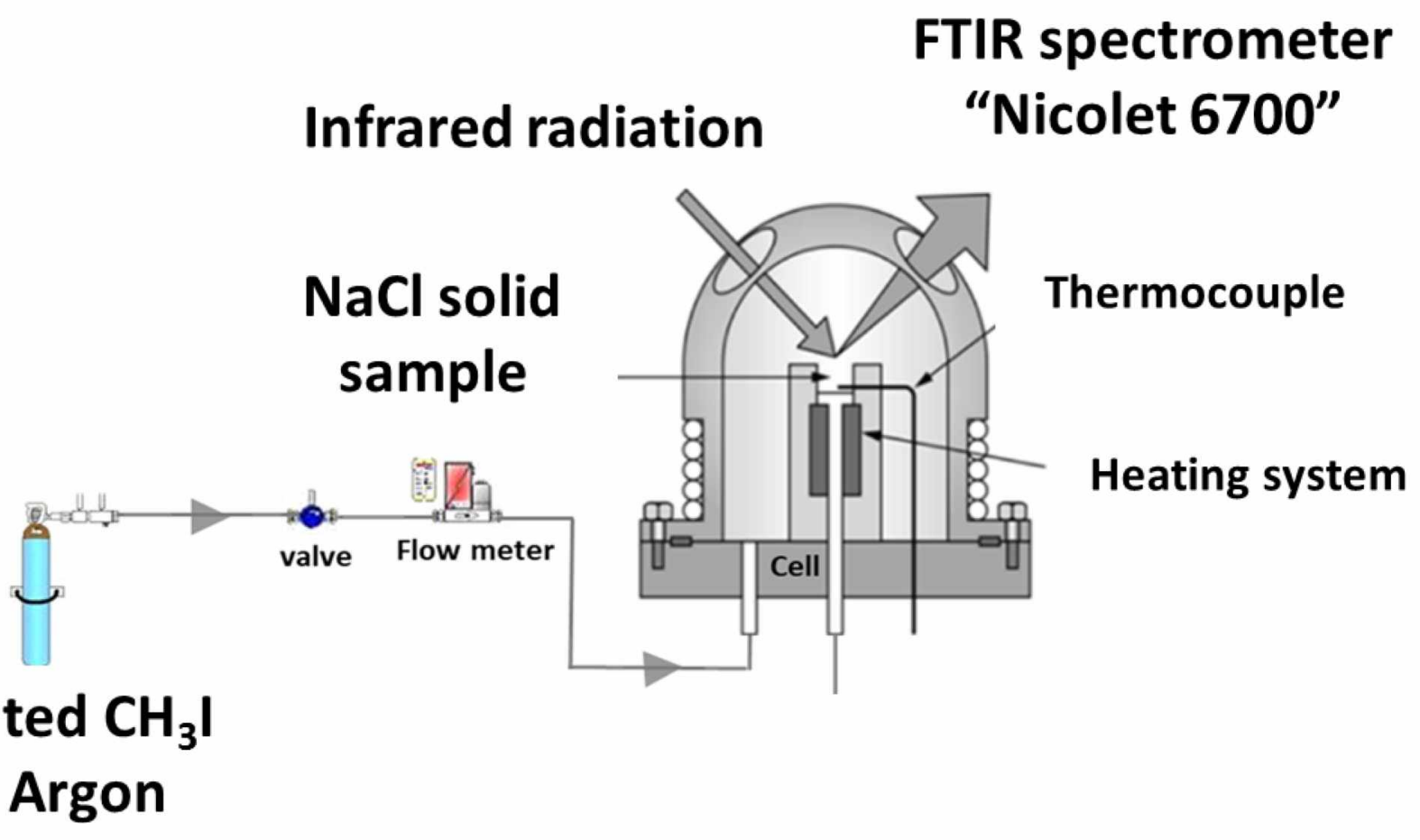

Experimental conditions

\begin{tabular}{|l|l|}
\hline Salt particles & $\mathrm{NaCl}$ (powder) \\
\hline$\left[\mathrm{CH}_{3} \mathrm{I}\right]$ & $1000 \mathrm{ppm}$ \\
\hline Total flow & $100 \mathrm{ml} / \mathrm{min}$ \\
\hline Temperature & Room Temperature \\
\hline Humidity & $\sim 10 \%$ at $20^{\circ} \mathrm{C}$ \\
\hline Pressure & $100 \mathrm{KPa}$ \\
\hline Total Exposure time & 5 hours \\
\hline
\end{tabular}

Spectral recording conditions

\begin{tabular}{|l|l|}
\hline Spectral range & 4000 to $700 \mathrm{~cm}^{-1}$ \\
\hline Resolution & $8 \mathrm{~cm}^{-1}$ \\
\hline Scans & 200 \\
\hline Time for each spectra & 8 minutes \\
\hline
\end{tabular}

The C-I stretching band at $572 \mathrm{~cm}^{-1}$ is not accessible, because the spectra is cut in the range below $700 \mathrm{~cm}^{-1}$. 


\section{Results: DRIFTS Spectra of $\mathrm{NaCl}$ when exposed to $\mathrm{CH}_{3} \mathrm{I}$}

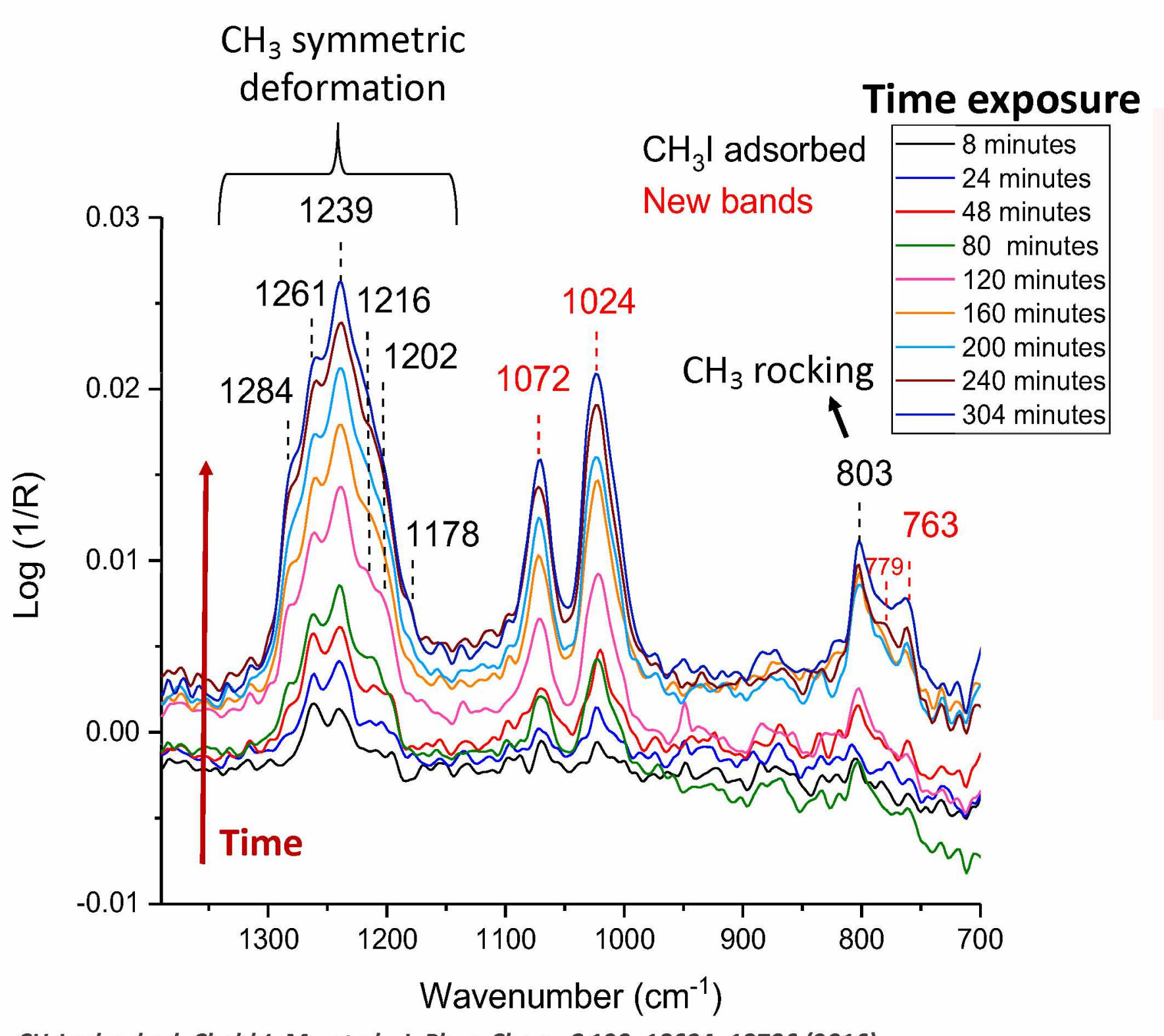

$\checkmark \mathrm{CH}_{3} \mathrm{l}$ adsorbed increases as a function of time.

$\checkmark$ New IR bands are observed due to $\mathrm{CH}_{3} \mathrm{I}$ interaction with $\mathrm{NaCl}$ - new product formation are observed.

$\checkmark$ No apparent desorption of $\mathrm{CH}_{3} \mathrm{l}$ and new bands are observed when the temperature increased or under $\mathrm{Ar}$ flow.

The kinetic for interaction between $\mathrm{CH}_{3} \mathrm{I}$ and $\mathrm{NaCl}$ follows a $1^{\text {st }}$ order law. 


\section{Theoretical study of the $\mathrm{CH}_{3}$ I microhydration}

\section{Objective}

Quantify $\mathrm{CH}_{3} \mathrm{I}$ interaction with water

$\rightarrow$ A first step to understand its chemistry

\section{Methods}

Quantum Mechanical calculations (Optimization+ Normal modes analysis)

$\rightarrow \omega B 97 X-D / a u g-c c-p V T Z(-P P)$ level of theory

$\rightarrow$ Standard reaction enthalpy $\left(\Delta_{r} H_{298 K}^{\circ}\right)$

$\rightarrow$ Standard Gibbs free reaction energy $\left(\Delta_{r} G_{298 K}^{\circ}\right)$

\section{Results}

Weak interaction, in agreement with Ito et. $_{\text {al. }}{ }^{1}$
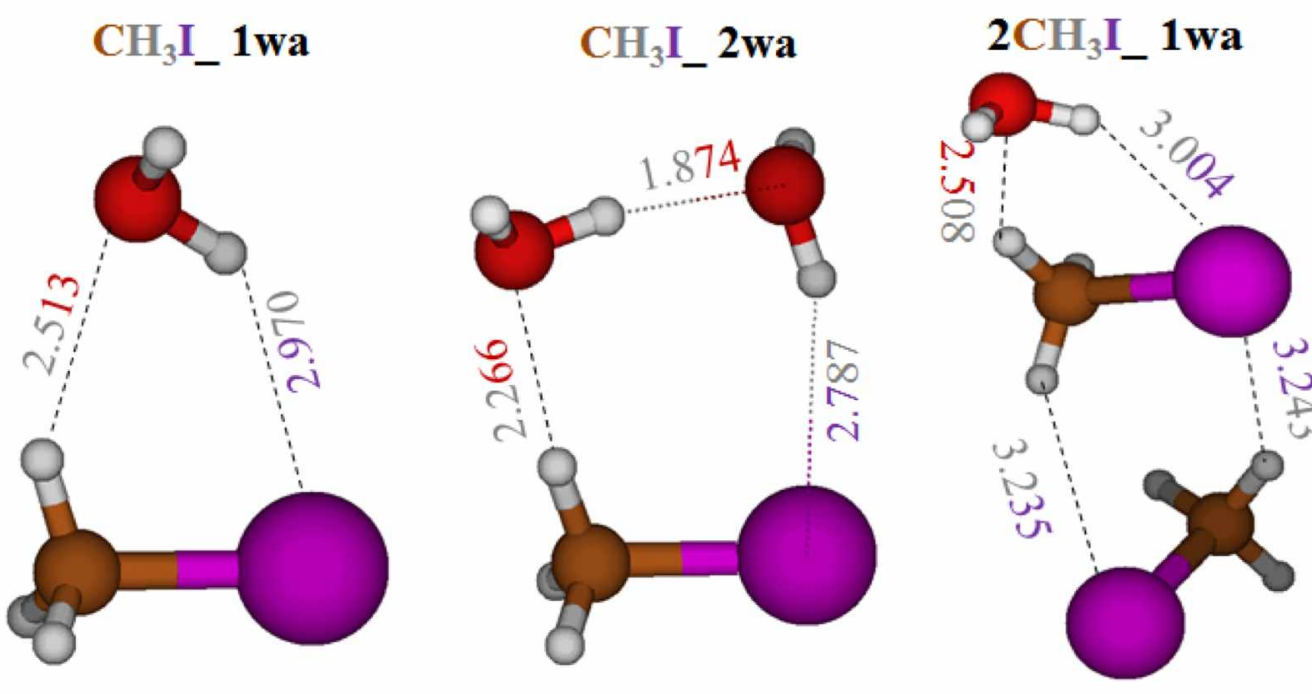

2 $\mathrm{CH}_{3} \mathrm{I} \_$2wa

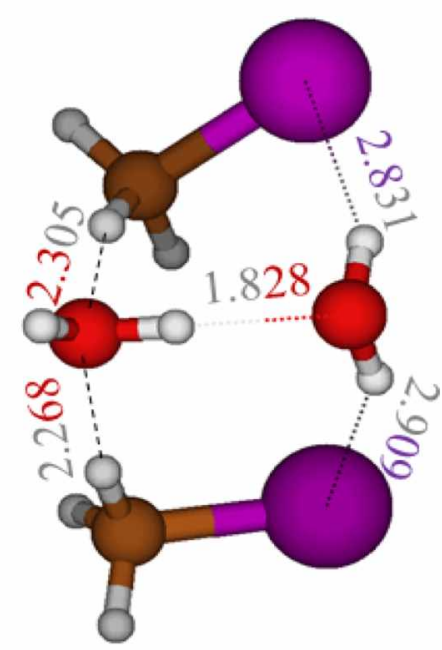

\begin{tabular}{|c|c|c|}
\hline Molecular complex & $\Delta_{\mathrm{r}} \mathrm{H}^{\circ}{ }_{298 \mathrm{~K}} / \mathrm{kJ} \mathrm{mol}^{-1}$ & $\Delta_{\mathrm{r}} \mathrm{G}^{\circ}{ }_{298 \mathrm{~K}} / \mathrm{kJ} \mathrm{mol}^{-1}$ \\
\hline $\mathrm{CH}_{3} \mathrm{I}+1 \mathrm{H}_{2} \mathrm{O}$ & -8.1 & 14.8 \\
\hline $\mathrm{CH}_{3} \mathrm{I}+2 \mathrm{H}_{2} \mathrm{O}$ & -33.1 & 25.5 \\
\hline $2 \mathrm{CH}_{3} \mathrm{I}+1 \mathrm{H}_{2} \mathrm{O}$ & -14.1 & 29.9 \\
\hline $2 \mathrm{CH}_{3} \mathrm{I}+2 \mathrm{H}_{2} \mathrm{O}$ & -53.3 & 43.5 \\
\hline
\end{tabular}

${ }^{1}$ Ito, F., et al. Chem. Phys. Lett.) 343, 185-191 (2001) 


\section{Ongoing: Interaction of $\mathrm{CH}_{3} \mathrm{I}$ with a model sea-salt particle}

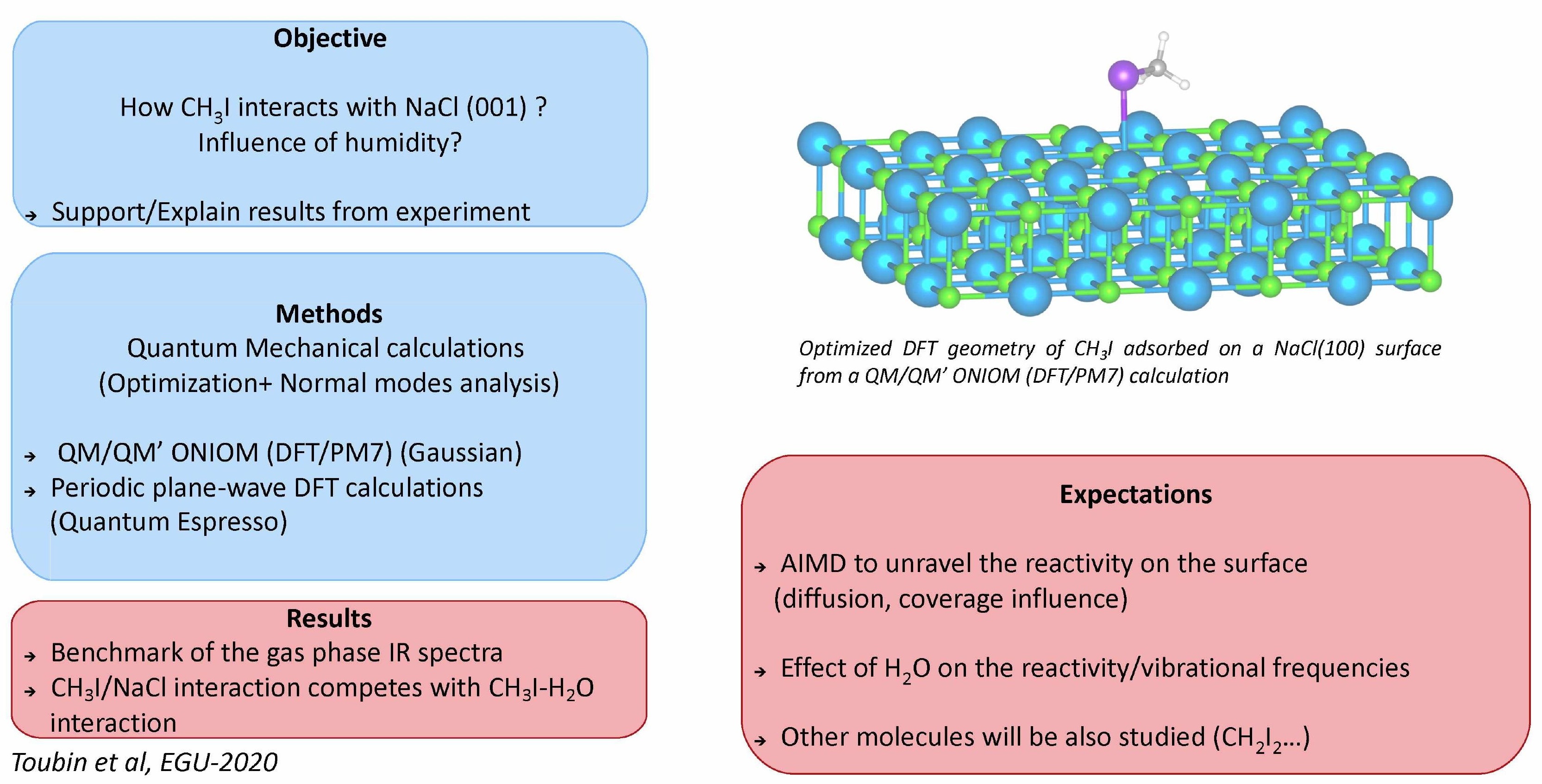

